\title{
Onboard Flight Termination System with Electronic Safe\&Arm Mechanism: A Rapid Solution for High Velocity Flight Vehicles
}

\author{
Muhittin AKTAŞ ${ }^{1}$, Celal ERTUĞRUL ${ }^{2}$, Celalettin KARAKUŞ ${ }^{2}$ \\ ${ }^{1}$ Roketsan Missiles Inc., Ankara/TURKEY, \\ maktas@roketsan.com.tr \\ ${ }^{2}$ Roketsan Missiles Inc., Ankara/TURKEY
}

\begin{abstract}
:
Flight Termination System is a key element for range safety during flight tests of high velocity flight vehicles, like missiles, sounding rockets or launch vehicles. Range safety regulations require immediate termination of errant flight either by an RF flight termination signal from ground stations or by an onboard autonomous decision mechanism. On the other hand, tight schedules of related projects put engineers under pressure.

An Onboard Flight Termination System with Electronic Safe\&Arm Mechanism developed by Roketsan and has completed three successful developmental flights. COTS products are used to shape the architecture of FTS which has been demonstrated to be a viable, reconfigurable and rapid solution for tight schedules of planned flight test activities. It was shown that FTS is capable of protecting life and property in event of an errant vehicle by terminating the flight. It is capable of replacing current humanin-the-loop systems or acting in parallel with them. FTS is configurable prior to flight with respect to mission specific rules set agreed upon by the range safety authority and the user to protect the public and assure mission success. This paper discusses the motivation for the project, describes the method of development and presents an overview of the architecture and the compatibility with RCC 319 standard.
\end{abstract}

Key words: Autonomous flight termination system, safe\&arm, range safety, flight vehicle, missile.

\section{Introduction and Purpose}

Flight Termination System is a key element for range safety during flight tests of high velocity flight vehicles, like missiles, sounding rockets or launch vehicles. Range safety regulations require immediate termination of errant flight either by an RF flight termination signal from ground stations or by an onboard autonomous decision mechanism. On the other hand, tight schedules of related projects put engineers under pressure. For flight testing of a flight vehicle that can endanger public safety, a flight termination system is required. Engineers had less than 5 months to design, produce, test, and integration phases. In order to meet this urgent need Geliştirme Amaçlı Uçuş Sonlandırma Sistemi (eng. Developmental Flight Termination System) (GAUSS) project is kicked off, also it satisfied the need successfully.
By proposed GAUSS design, four different flight termination methods are realized reliably, three of them are autonomous; termination upon ground command, termination after predefined flight time elapsed, termination by loss of RF link with ground station and termination due to low voltage of batteries.

In this article, electronic parts including RF components that shape GAUSS architecture is provided with main technical parameters. Also operational sequence and working principles of GAUSS is explained. Finally applied tests for qualification and compatibility with RCC-319 standard are discussed.

\section{System Architecture}

GAUSS is composed of an antenna set, a receiver box, a decision unit and a battery pack. Onboard antenna set has three antennas those collect the RF signal of interest and deliver to receiver box. Receiver box includes two identical RF receivers for demodulating 
commands sent from ground station and interface to the decision unit. Decision unit, as its name implies, determines if conditions for flight termination are occurred or not. Mainly, It interprets message delivered from receiver box, counts flight time, controls safe\&arm switches according current status of flight, shapes the activation signal of pyrotechnics and forms telemetry data. Finally battery pack provides the power for electronic units and to activate pyrotechnics. All units of GAUSS designed fully redundant in order to meet 0.999 reliability requirement. Details of each unit are explained in related subsections. Functional architecture of GAUSS is shown below. (see Fig. 1).

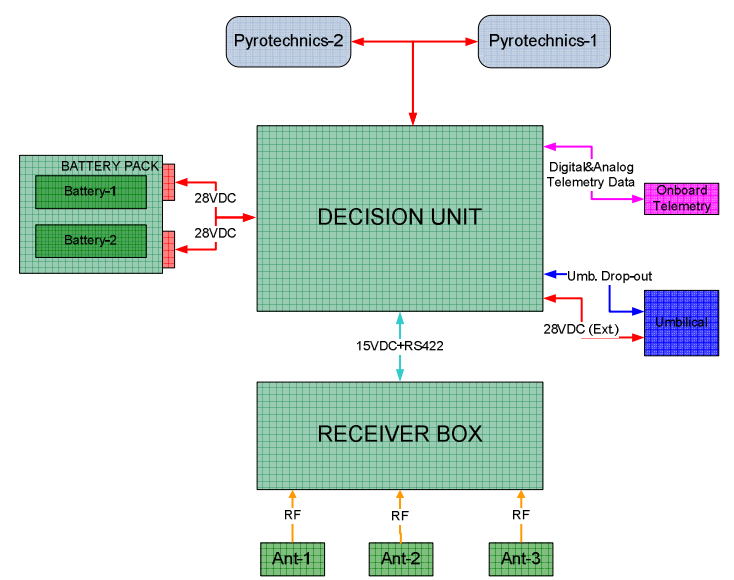

Fig. 1. GAUSS Architecture

\section{Decision Unit}

Decision Unit (DU) is responsible for managing flight termination system and acts as "brain" of it. Demodulated RF messages are delivered to and interpreted in DU. DU completes the actions commanded in messages received from Receiver Box. These actions include switching from external power to battery power, forcing start of the flight time and flight termination. It gives flight termination decision after predefined flight time elapses, RF link loss with ground station or low battery voltage. Dimensions of DU are $200 \mathrm{~mm} \times 150 \mathrm{~mm} \times 100 \mathrm{~mm}$ (Length $\mathrm{x}$ Width $x$ Height) without mounting fixtures. 3D model of Decision Unit is shown below. (see Fig. 2)

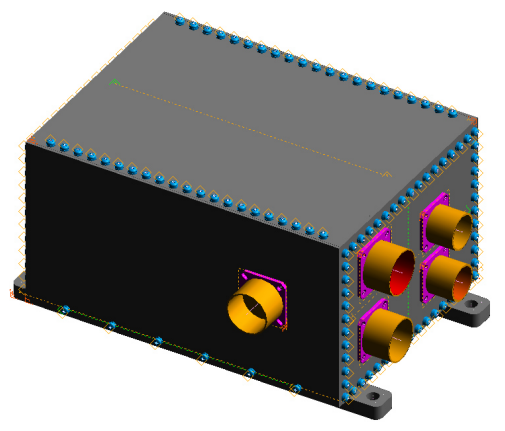

Fig. 2. 3D Model of Decision Unit
Decision unit consists of three electronic cards of two kinds, namely two Termination Control Cards and a Power Management \& Telemetry Card. (see Fig. 3). Both cards are based on non-volatile FPGAs, that has $50 \mathrm{~K}$ system gates, 1584 logic cells and $1 \mathrm{M}$ flash.

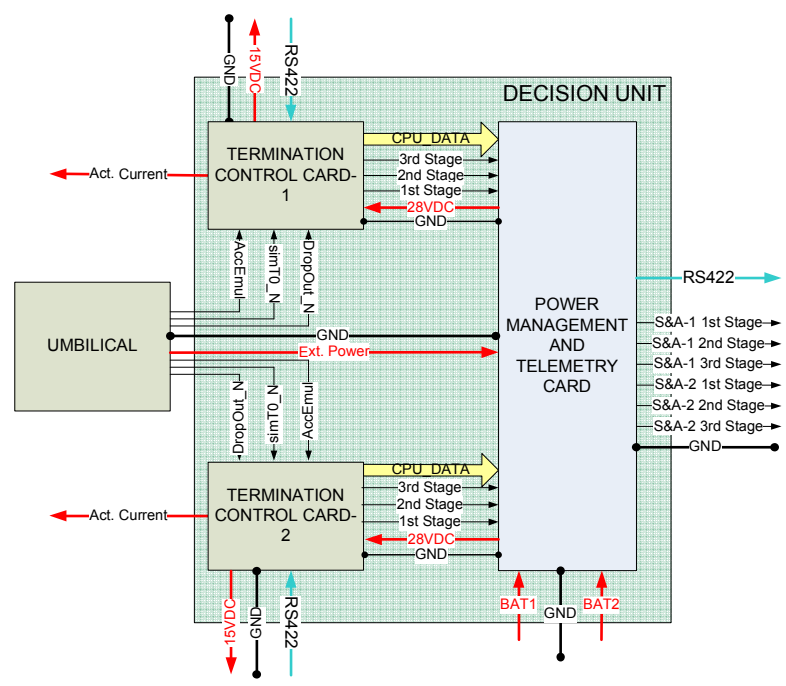

Fig. 3. Decision Unit Architecture

Power Management \& Telemetry Card (PMTC) interfaces Decision Unit to External Power, supplying from ground station before flight and Battery Pack that used during flight. It provides capability of switching between external power and battery power when commanded. Possible noise on power line is filtered out by using Synqor's military grade, quarter brick passive filters and delivered to both PMTC's own and Termination Control Cards (TCC). Batteries voltages from two batteries are paralleled before distributing them to other components on this card. Battery voltages and currents draining from batteries are measured before paralleling using two industrial supervisor chips. PMTC also converts 28 VDC battery voltage to secondary voltages as its onboard FPGA and other components needs.

PMTC is responsible for packing and delivering telemetry data of GAUSS. Digital data from Termination Control Cards (TCC) and measured voltages/currents from supervisor chips are collected and digital telemetry frame are created by FPGA. On PMTC, all UART communications, including communication between PMTC and TCCs, Decision Unit and OTE is managed by FPGA via RS422 interface using MAX 490 chips. Additionally, PMTC converts analog telemetry signals to $10 \mathrm{~V}$ range and delivers them to OTE. Digital and analog telemetry data are shown below. (see Tab. 1) 
Tab. 1: Telemetry Data

\begin{tabular}{|c|c|c|}
\hline No & Data & Source \\
\hline 1 & Battery Voltages & PMTC \\
\hline 2 & Battery Currents & PMTC \\
\hline 3 & Squib Test Results & TCC \\
\hline 4 & Termination Flag & TCC \\
\hline 5 & Drop-out Monitor & TCC \\
\hline 6 & Mode & TCC \\
\hline 7 & Station ID & TCC \\
\hline 8 & RF Message & TCC \\
\hline 9 & RF Error Count & TCC \\
\hline 10 & FPGA Times & TCC \\
\hline 11 & CPLD Times & TCC \\
\hline 12 & IMU Supply Volt. & TCC \\
\hline 13 & IMU Gyro Volt. X & TCC \\
\hline 14 & IMU Gyro Volt. Y & TCC \\
\hline 15 & IMU Gyro Volt. Z & TCC \\
\hline 16 & IMU Acc. Volt. X & TCC \\
\hline 17 & IMU Acc. Volt. Y & TCC \\
\hline 18 & IMU Acc. Volt. Z & TCC \\
\hline 19 & Arming Status. & TCC \\
\hline
\end{tabular}

Decision Unit has two identical TCCs. Each TCC works independently, i.e. each TCC gives power to different receivers and controls different pyrotechnics in order to prevent single point failure. TCC converts 28 VDC to secondary voltages as PMTC, it also produces 5 VDC for IMU chip on it and 15 VDC for RF Receiver. Again, same non-volatile FPGA is used on TCC in order to manage tasks including serial communications, timing and logic decisions.

TCCs have their own electronic safe \& arm circuits on them. Each safe \& arm circuit has three safety stages on it realized by twin redundant hard relays. First stage of relays is closed by a predetermined acceleration value in flight direction ( $1^{\text {st }}$ group in Fig. 4). Acceleration measurements in three-directions are made by single axis, $200 \mathrm{mV} / \mathrm{g}$ sensitive accelerometers. Here, it is good to note that first safety stage can be by-passed by emulated signals sent from umbilical or via RF commands. This option is added for ground testing of GAUSS. It also helps reliable flight termination action in case of projected acceleration is not measured by accelerometers because of erroneous launch or a failure in accelerometers.

Second stage is closed when safe separation time passes ( $3^{\text {rd }}$ group in Fig. 4). Safe separation time is the duration that test item is far enough from launching point not damage launch site and launching crew. From analyses of related flight vehicle and test range specifications, safe separation time is predefined as 5 seconds. Safe separation time is counted by FPGA.

Third stage is closed when termination command received, RF link loss exceeds predefined duration, completion of predefined flight time or low voltage of batteries $\left(2^{\text {nd }}\right.$ group in Fig. 4). Flight time is counted by both FPGA and CPLD's in order to increase reliability. Three low power CPLD's that can support internal clock frequency rates up to $300 \mathrm{MHz}$ are used for this task. FPGA and CPLD's create totally 8 signals that each of them is enough to close third stage safety relays. Once closed, all relays are latched. After all safety stages are closed TCC shall deliver several Amperes of current for duration that guarantees reliable activation pyrotechnics. This is obtained by direct connection of the batteries and pyrotechnics after all safety relays are closed. Safety stages and default positions of relays before flight are shown below. Note that two poles of pyrotechnic devices are short circuited until safe separation time is elapsed. (see Fig. 4)

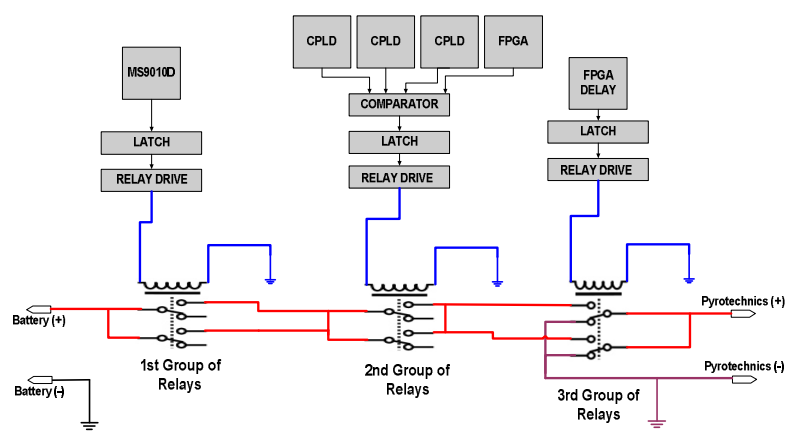

Fig. 4. Electronic Safe and Arm Device

Note that $3^{\text {rd }}$ group of relays that maintains second stage of safety (safe separation time) are the last circle of the activation chain. That's because of positive and negative poles of pyrotechnics line are intended to be in short circuit state until the flight vehicle is in a safe distance for launch site. After safe separation time elapses, these lines are switches to readyto -activate position.

All parameters for controlling safety stages (magnitude and direction of acceleration, safe separation and flight times, RF link loss time for 
flight termination) are programmable via JTAG interface of FPGA.

Both TCCs are equipped with, six degrees of freedom inertial sensor chips for experimental purposes. Acceleration and gyro measurements obtained from these chips are telemetered. This data is not used actively for onboard decision mechanisms. It has provided a comparable data with primary IMU of flight vehicle. In the future, this data also can be used for implementing onboard decision mechanisms to terminate flight by calculating instantaneous impact points.

Each TCC is capable of testing related pyrotechnic activation line's continuity. Test is realized by using FPGA.

Finally, telemetry data of each TCC (see Tab. 1 ) is packed by FPGA and sent to PMTC using RS 422 interface. Analog signal lines indicating statuses of safety stages are also delivered to PMTC.

\section{Receiver Box}

Receiver Box is used to receive and demodulate RF commands those are sent from ground station. RF signal collected by three antennas are delivered to Receiver Box. Inside receiver box, three antenna inputs are combined and then divided again to two identical receiver units using COTS combiner and divider units. They have maximum $0.3 \mathrm{~dB}$ insertion loss, minimum $19 \mathrm{~dB}$ isolation and maximum 1.35:1 VSWR.

Receiver units are also COTS airborne products that work at $L$ band with nearly -80 $\mathrm{dBm}$ sensitivity, pricing around $£ 16000$ for each. Receiver sensitivity is selected according to link budget calculations using theoretical trajectory of flight vehicle that is to be tested. For reliable operation, $12 \mathrm{~dB}$ link margin is objected. Center frequency is programmable with $0.1 \mathrm{MHz}$ steps. Bitrate of input signal is also programmable between 9600 and 115200 bps. Receivers are capable of outputting demodulated data via RS 422 interface. They are fed with 15 VDC supplied from DU. Interfaces and 3D model of Receiver box is shown below. (see Fig. 5 and Fig. 6) Dimensions of receiver box are $210 \mathrm{~mm} \times 75$ $\mathrm{mm} \times 160 \mathrm{~mm}$ (Length $\times$ Width $\times$ Height) without mounting fixtures.

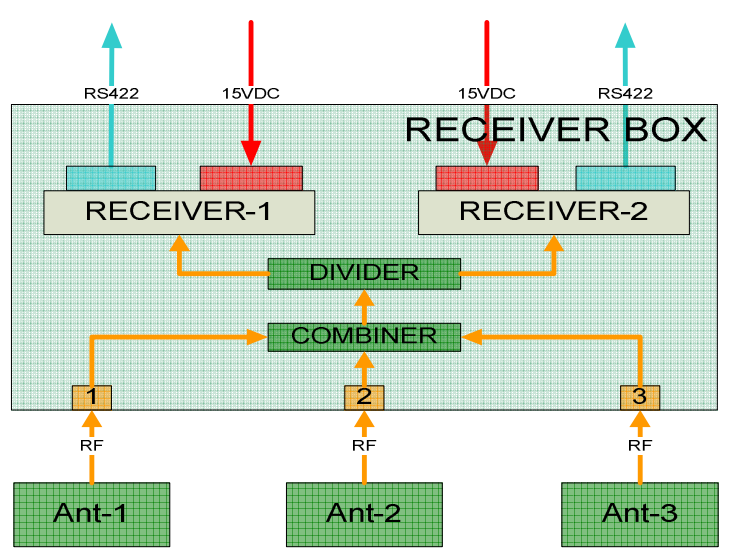

Fig. 5. Receiver Box Connection Diagram
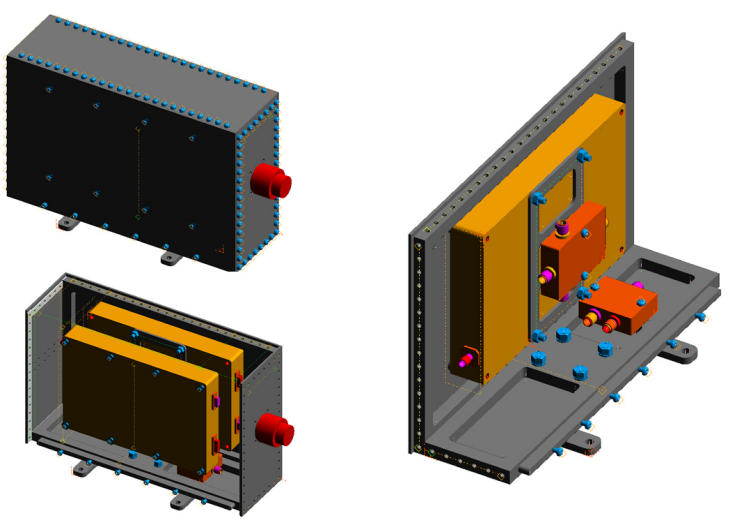

Fig. 6. 3D Model of Receiver Box

\section{Antenna Set}

Three antennas form onboard Antenna Set. Blade type antennas are used onboard antennas. (see Fig. 7)

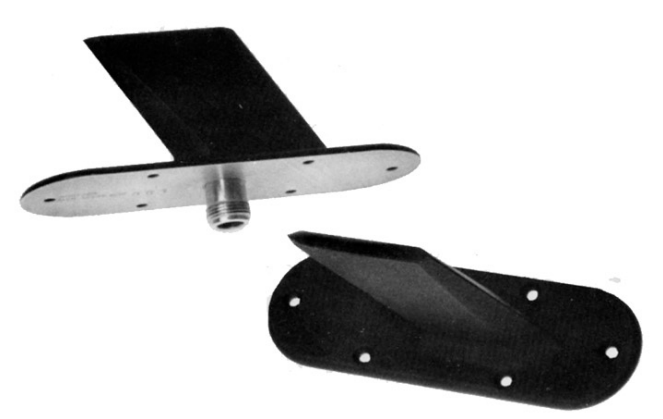

Fig. 7. Typical Blade Antenna

Blade antenna is about $40 \mathrm{~mm}$ height and 25 grams weight. These antennas are worse than conformal antennas by means of aerodynamics but performance/procurement duration was suitable for the project requirements so they are selected to use onboard. They have linear polarization and maximum 1.5:1 VSWR over operating band of RF receivers. Antennas are 
connected to receiver box with SMA connectors.

Antenna has hemispherical pattern. It has omnidirectional pattern in azimuth with $0 \mathrm{dBi}$ gain. In elevation, it has minimum $-5 \mathrm{dBi}$ gain between $90^{\circ}$ and $-90^{\circ}$ including $0^{\circ}$ bore sight of the antenna. In order to shape an omnidirectional pattern around the flight vehicle three antennas are mounted with $120^{\circ}$ separation between each other.

\section{Battery Pack}

Battery Pack includes two identical Li-ion batteries each can supply current for components of two channels with $150 \%$ margin under 30 Volts. Each battery weighs around 1.5 $\mathrm{kg}$. In order to mount the battery to the flight vehicle a unique metallic fixture was used (see Fig. 8).

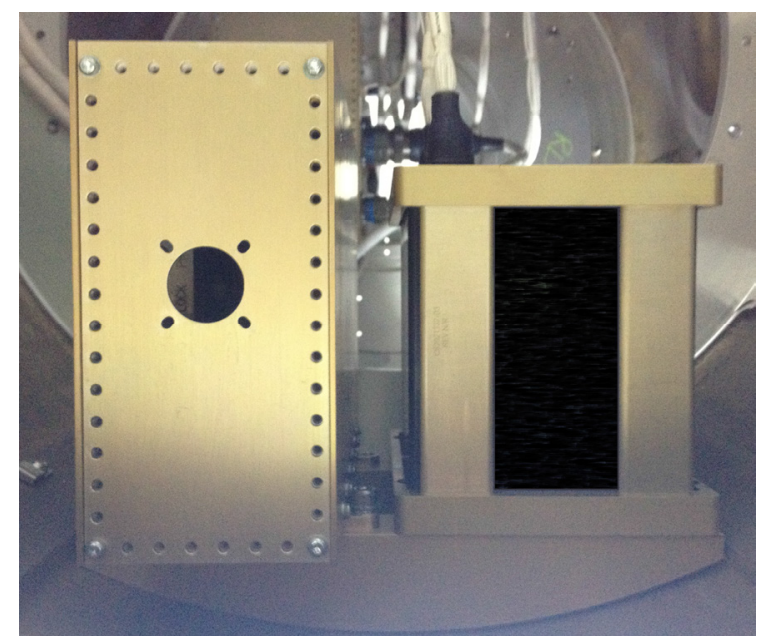

Fig. 8. Li-Ion Battery in Its Metallic Case with a Dummy Receiver Box mounted on Flight Vehicle

\section{Operational Sequence}

GAUSS has 5 different operating modes. After GAUSS is powered-on, it checks pyrotechnics lines continuity. If it is detected that two pyrotechnics are connected properly, GAUSS switches to "Idle Mode (IM)" and waits for RF commands. In parallel it starts to measure battery voltages, IMU outputs and send telemetry data automatically at each $10 \mathrm{~ms}$. If pyrotechnics lines' continuity check is not passed GAUSS switches to "Emergency Stop Mode (ESM)". In ESM, power is switched to external power, all safety relays are switched to open state except the $3^{\text {rd }}$ group. $3^{\text {rd }}$ group relays are switched such position that positive and negative poles of pyrotechnics line are short circuit state in order to prevent an unintended activation of pyrotechnics. Flowchart of opening sequence of GAUSS is shown below (see Fig. 9).

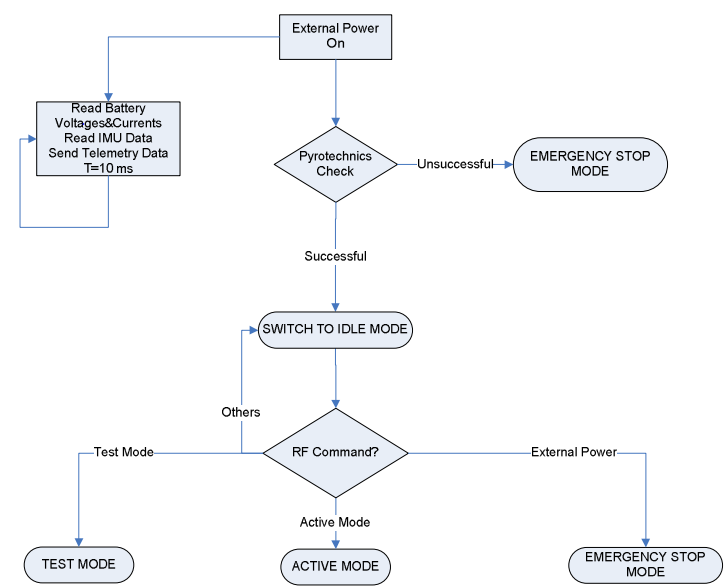

Fig. 9. Opening Sequence of GAUSS

In order to initiate launching sequence, GAUSS should be switched to "Active Mode (AM)". In AM, GAUSS waits for RF command to switch battery power. Eventually, it checks if flight is started, i.e. umbilical connector is still connected or not. In case of umbilical drop-out before Internal Power Command is received, GAUSS switches to ESM.

After Internal Power Command is received, GAUSS switches to Battery Power and starts to wait for umbilical drop-out. After umbilical dropout sensed, GAUSS switches to "Flight Mode (FM)". After launch, it is expected that flight vehicle's movement shall cause $1^{\text {st }}$ stage safety relays to close. In case of a failure in umbilical continuity check line, in accelerometers or an unexpected launch movement (e.g. lower acceleration values than expected) GAUSS can be switched to FM manually by an RF command, namely Flight Mode command. When this command is received, $1^{\text {st }}$ stage of safety is by-passed and related relays are forced to close.

FPGAs and CPLDs start to count flight time with $10 \mathrm{~ms}$ resolution. This duration is also one processing cycle of GAUSS. In FM, predefined flight times for safe separation distance and termination are checked periodically in every cycle. After safe separation time is elapsed, $3^{\text {rd }}$ group of relays are switched to activation state. Completion of predefined flight time causes termination action.

During normal flight, Flight Mode or Safe commands with frame counters in them are transmitted periodically from flight termination ground station. GAUSS checks for a new RF command at every cycle. If RF link between onboard unit and ground station is lost for a predefined duration GAUSS switches to "Termination Mode (TM)". Additionally, if RF Arm command is received in any portion of flight GAUSS also switches to TM. Only one 
successful command is decided to be enough for starting termination action upon receiving Arm command to minimize potential hazard in risk, in case of a bad RF link. In order to prevent demodulation and decoding errors and receiving a "false alarm" Arm command, Arm command is selected to have maximum Hamming distance from other commands. Additionally, in every frame, command words are repeated and valid command is selected by voting.

In TM, safe separation duration is checked first. If it is already elapsed activating signal is delivered to pyrotechnics by closing $2^{\text {nd }}$ group of relays for $300 \mathrm{~ms}$. Activation signal is repeated to ensure success of flight termination in case of misfire. Operational flowchart of GAUSS during flight is shown below. (see Fig.10)

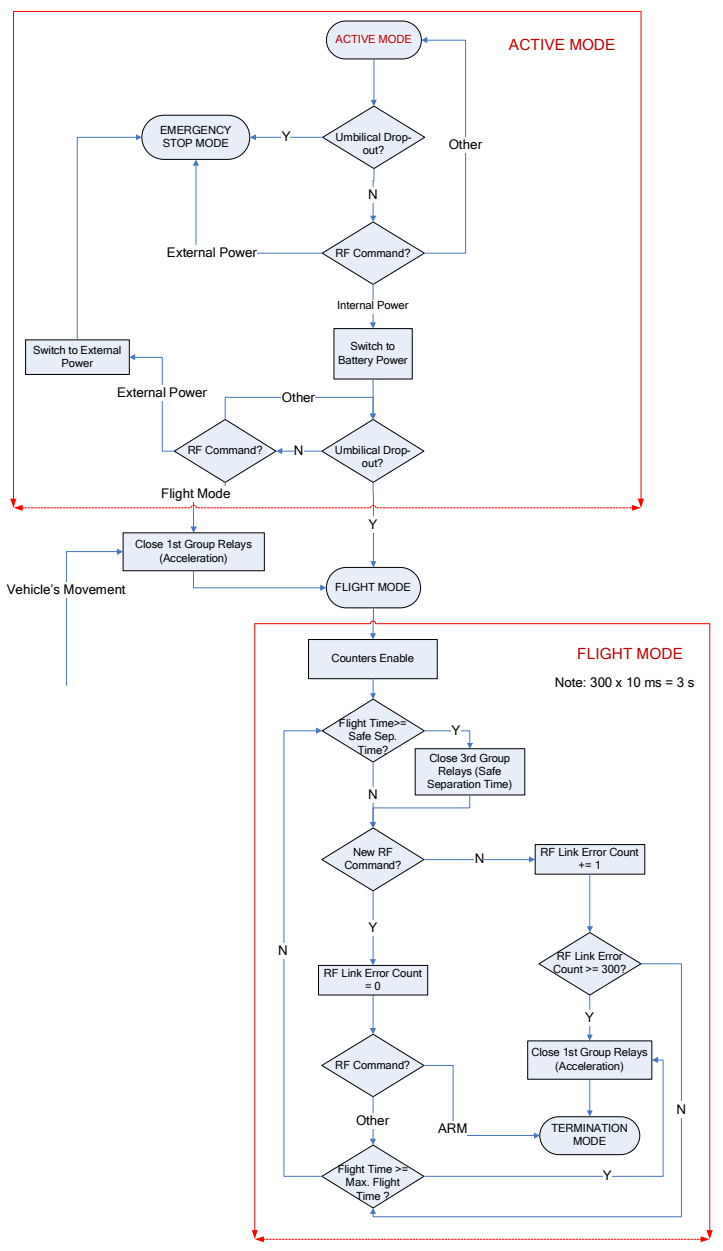

Fig. 10. Flight Sequence of GAUSS

\section{Cabling and Harness}

All signals of flight termination system are carried with redundant lines. Connectors and cables are selected among military grade components.

\section{Environmental Requirements and Qualification}

GAUSS is designed to operate in harsh environmental conditions, such as high temperature (up to $100^{\circ} \mathrm{C}$ in a couple of minutes), severe vibration (reaching $15 \mathrm{gRMS}$ ) and low pressure/high altitude (corresponding a few dozens of kilometers). Mechanical design, PCB design and component (avionic enclosure materials, electronic components, COTS products and harness components) selection is made according to these harsh environmental conditions. Fixtures those are used to integrate GAUSS to flight vehicle are designed not to amplify frequency modes of both flight vehicle and GAUSS. Also, a reliability analysis using dedicated software is conducted.

Operability under environmental conditions is verified through ground tests. Mainly, temperature, random vibration, acceleration and low pressure/high altitude tests are conducted to simulate flight environment. Additionally, in order to eliminate workmanship defects and faulty components during producing phase, environmental stress screening (ESS) tests are applied to every unit according to MIL-HDBK-344A. ESS also provides burn-in phase to electronic equipment; it helps to reach middle portion of the bathtub curve of product lifecycle-probability of failure (portion with least probability of failure) graph.

\section{Ground Station}

Ground Station of proposed design is composed of three main items which are RF Data Transmission System, directional antenna system, and a laptop-based control system. Two independent ground systems are used at different locations of test site for full redundancy.

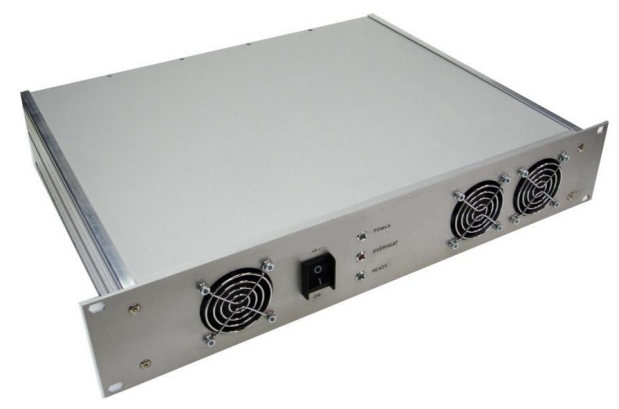

Fig. 11. Typical Data Transmission System

The Data Transmission System which has maximum $40 \mathrm{~W}$ RF power output can be controlled remotely by an external computer via RS232 interface. This feature offers possibility of maintenance and easy update to the system. remotely. The FTS control software features a graphical user interface (GUI) that can be easily 
customized to suit different requirements. The control unit has a remote control interface which sends the required commands that can be selected from GUI.

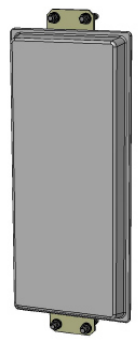

Fig. 12 Typical Directional Antenna

COTS directional RHCP antenna is used as the ground antenna (see Fig.12). Because the tight schedule and need to provide a quick solution, antenna system does not include a tracking controller unit. In order to achieve high performance, optimal FTS ground system location is determined regarding the azimuth and elevation angle variation relative to the flight trajectory. Antenna beam widths and placements are selected according to worst case azimuth and elevation angles with respect to predefined trajectory.

\section{RF Message Format}

RF message of GAUSS consists of six blocks of the data including a frame synchronization word, ground station range ID number, transmission system ID number, counter for each command sent and Cylic Redundancy Check (CRC) block as shown below. (see Tab. 2.)

Message format is chosen to maintain safe operation and minimum processing effort. Time counter is used for determining the time of the link loss. The format and index of the message can be updated easily via GUI interface.

Tab. 2: Message Format

\begin{tabular}{|c|c|c|c|c|c|}
\hline $\begin{array}{c}\text { Frame } \\
\text { Synch }\end{array}$ & $\begin{array}{c}\text { Range } \\
\text { ID }\end{array}$ & $\begin{array}{c}\text { TX } \\
\text { ID }\end{array}$ & Counter & Command & CRC \\
\hline
\end{tabular}

\section{RF Link Margin}

A radio propagation model (see Fig.14) and link analysis was conducted for the GAUSS. The RF link analysis includes path losses due to rain, fog, plume attenuation, other possible attenuation factors; ground system RF characteristics, onboard transmission characteristics and vehicle trajectory. The parameters used in the analysis are given below. (See Fig. 14 and Tab. 3). It is guaranteed to maintain $12 \mathrm{~dB}$ link margin in order to ensure reliable operation, attenuation values are exaggerated.

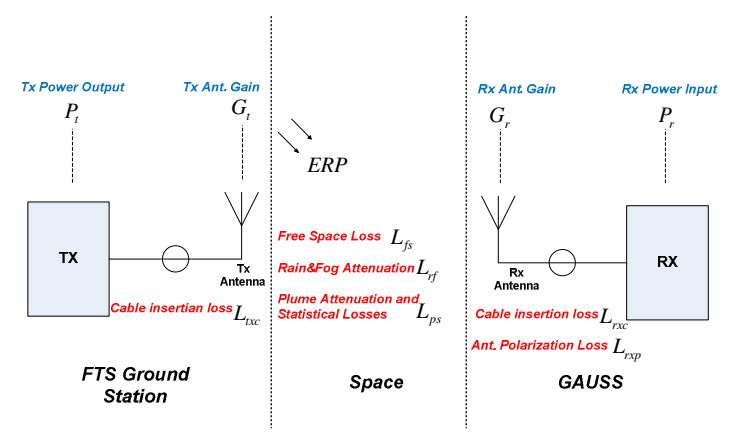

Fig. 14 Radio Propogation Model

Tab. 3: Parameters Used in RF Link Margin Calculation

\begin{tabular}{|c|l|}
\hline S/N & \multicolumn{1}{|c|}{ Parameter } \\
\hline 1 & TX Output Power $\left(P_{t}\right)$ \\
\hline 2 & TX Cable Loss $\left(L_{t x c}\right)$ \\
\hline 3 & TX Antenna Gain $\left(G_{t}\right)$ \\
\hline 4 & ERP \\
\hline 5 & Frequency $(f)$ \\
\hline 6 & Range $(r)$ \\
\hline 7 & Free Space Loss $\left(L_{f s}\right)$ \\
\hline 8 & $\begin{array}{l}\text { Plume Attenuation and Statistical } \\
\text { Losses }\left(L_{p s}\right)\end{array}$ \\
\hline 9 & Rain and Fog Attenuation $\left(L_{r f}\right)$ \\
\hline 10 & Total Loss in Space $\left(L_{f s}\right)$ \\
\hline 11 & RX Antenna Gain $\left(G_{r}\right)$ \\
\hline 12 & $\begin{array}{l}\text { RX Antenna Polarization Loss } \\
\left(L_{r x p}\right)\end{array}$ \\
\hline 13 & RX Cable Loss $\left(L_{r x c}\right)$ \\
\hline 14 & Received Power $\left(P_{r}\right)$ \\
\hline 15 & Receiver Sensitivity \\
\hline 16 & Link Margin \\
\hline & \\
\hline 10
\end{tabular}

\section{Compatibility with RCC 319}

During design phase of GAUSS, compatibility with basic requirements of RCC 319-10 (319-14 version was not released yet) is targeted, like redundancy of safety critical components that will affect desired termination action 
(Termination Control Cards, batteries, receivers, cables and pyrotechnics), three stages of safety built-in-test capability and component derating criteria. On the other hand, some constraints restricted design team to stay incompatible with flight termination systems' international standard. Such requirements are frequency band, modulation technique or crossstrapping of fail-safe actions. [1].

Main difference between GAUSS design and RCC 319 is operating band and modulation technique. UHF band and frequency modulation of analog signals are proposed in standard. However, that is not compatible with standard yet useful and fastest solution to satisfy project needs are selected.

A fail-safe system shall generate Arm and Terminate outputs when it has been enabled and any specified fail-safe condition occurs [1]. Both loss-of-power and loss-of-command-link fail-safe measures are enabled in GAUSS design. In case of a failure in one of two redundant channels of flight termination system, in order to continue operation with fault-free channel, two channels should be crossstrapped with a termination inhibit logic. However, since design and testing phase of GAUSS is very short in time, cross-strapping of redundant channels is not implied. In order to maintain to continue the operation when one of batteries fails, powers of two batteries are paralleled in DU.

As ground antenna used in this project has no auto-track capability, a conservational approach for loss-of-command-link is executed. If one of two Termination Cards doesn't receive a new message from RF receivers for a predetermined time flight is terminated. Continuing the flight with one receiver is not considered as a reliable operation. Implying cross-strapping logic proposed in RCC 319 shall maintain true fail-safe measure.

Another incompatibility with RCC 319 is; RCC requires flight termination receivers' to have $107 \mathrm{dBm}$ guaranteed sensitivity at least. However, due to tight schedule of project, purchasable COTS receiver-transmitter couple that can provide safe link margin in test scenario with shortest lead time is preferred.

\section{Summary}

GAUSS is proposed and demonstrated successfully in flight tests as a maybe not cost efficient but saving-the-day solution. It satisfies key requirements of RCC-319 standard. It is hoped that this design shall maintain a baseline for a flight termination system that is fully compatible with range safe community's requirements. Improving and adding more intelligence to autonomous functions, truly implementing fail-safe inhibits and more reliable radio links are taken as future design goals.

\section{References}

[1] Range Commanders Council, Range Safety Group, Document 319-10 Flight Termination Systems Commonality Standard

[2] Department of Defense, Military Handbook, MILHDBK-344A Environmental Stress Screening of Electronic Equipment. doi: 16 August 1993 